\title{
Microcavity polariton linewidths in the weak-disorder regime
}

Borri, Paola; Langbein, Wolfgang Werner; Woggon, U.; Jensen, Jacob Riis; Hvam, Jørn Märcher

Published in:

Physical Review B (Condensed Matter and Materials Physics)

Link to article, DOI:

10.1103/PhysRevB.63.035307

Publication date:

2000

Document Version

Publisher's PDF, also known as Version of record

Link back to DTU Orbit

Citation (APA):

Borri, P., Langbein, W. W., Woggon, U., Jensen, J. R., \& Hvam, J. M. (2000). Microcavity polariton linewidths in the weak-disorder regime. Physical Review B (Condensed Matter and Materials Physics), 63(3), [035307]. https://doi.org/10.1103/PhysRevB.63.035307

\section{General rights}

Copyright and moral rights for the publications made accessible in the public portal are retained by the authors and/or other copyright owners and it is a condition of accessing publications that users recognise and abide by the legal requirements associated with these rights.

- Users may download and print one copy of any publication from the public portal for the purpose of private study or research.

- You may not further distribute the material or use it for any profit-making activity or commercial gain

- You may freely distribute the URL identifying the publication in the public portal 


\title{
Microcavity polariton linewidths in the weak-disorder regime
}

\author{
P. Borri, W. Langbein, and U. Woggon \\ Experimentelle Physik EIIb, Universität Dortmund, Otto-Hahn Strasse 4, 44227 Dortmund, Germany \\ J. R. Jensen and J. M. Hvam \\ Research Center COM, The Technical University of Denmark, Building 349, DK-2800 Kgs. Lyngby, Denmark
} (Received 14 June 2000; revised manuscript received 11 October 2000; published 27 December 2000)

\begin{abstract}
Polariton linewidths have been measured in a series of high-quality microcavities with different excitonic inhomogeneous broadening in the weak-disorder regime. We show experimentally that the influence of the disorder on the polariton linewidths is canceled when the polariton energies are far in the tail of the excitonic absorption. The measured linewidths are quantitatively compared with an estimation using the measured excitonic absorption spectrum of the bare quantum wells, and good agreement is found.
\end{abstract}

DOI: 10.1103/PhysRevB.63.035307

PACS number(s): 78.66. $-\mathrm{w}, 78.66 . \mathrm{Fd}, 71.36 .+\mathrm{c}$

The role of static disorder in the linewidth of cavity polaritons has been intensively discussed in recent years, experimentally and theoretically. In 1996, Whittaker et al. ${ }^{1}$ reported reflectivity spectra measured on a GaAs semiconductor microcavity (MC) containing three InGaAs quantum wells (QW's). They observed that when the cavity mode is in resonance with the bare heavy-hole ( $\mathrm{HH})$ exciton transition the linewidth of the lower polariton (LP) is smaller than expected by averaging the bare exciton and cavity linewidths. Moreover, the lower-polariton linewidth is narrower than that of the upper polariton. They explained the subaverage broadening with a scaling theory based on a motional narrowing argument: When a quantum particle of finite size moves in a disordered potential with correlation length smaller in size, the spectral linewidth is reduced compared to that of a classical particle due to spatial averaging over the disorder. However, their model, which neglected interbranch scattering and nonparabolicity of the polariton dispersion, failed in explaining the different linewidths of the lower and upper branches near resonance. In 1997, Savona et al. ${ }^{2}$ proposed a one-dimensional microscopic model of disordered quantum wells embedded in a microcavity where excitonphoton coupling and exciton-disorder interaction are treated nonperturbatively. This model was able to reproduce both the subaverage broadening due to motional narrowing, and the larger linewidth in the upper polariton due to additional interbranch scattering. In 1998, Ell et al. ${ }^{3}$ showed that all these features were reproduced by using the measured optical absorption of the bare QW together with linear dispersion theory. They concluded that the disorder-averaged excitonic response in the bare QW fully determines the optical properties of the well embedded in a microcavity, and in particular that the asymmetric line shape of an inhomogeneously broadened exciton absorption ${ }^{4}$ is responsible for the narrower LP linewidth compared to that of the upper polariton. At the same time Whittaker ${ }^{5}$ proposed a unified picture where numerical calculations using a microscopic twodimensional model were in good agreement with a simpler model describing the polariton to exciton scattering in terms of an absorption picture. In this model, the polariton linewidth is given by the photon lifetime due to escape through the mirrors and by the loss of photons due to absorption into exciton states. In other words, the photonic fraction of the upper and lower polaritons is resonant with states in the tails of the exciton absorption that are in the weak-coupling regime and simply act as a source of absorption in the cavity. Therefore, in this model the difference between the widths of the two polariton branches is a consequence of the asymmetry of the inhomogeneously broadened exciton line shape, with larger absorption on the high-energy side. ${ }^{4}$ This absorption model is thus a simplified version of the model of Ell et al., ${ }^{3}$ but in essence also agrees with the suggestion of Savona et $a .^{2}{ }^{2}$ that the large width of the upper branch is caused by scattering of polaritons into higher-momentum exciton states. It is emphasized by Whittaker ${ }^{5}$ that this simple model is justified because motional narrowing effectively eliminates the contribution to the linewidth due to disorderinduced scattering between low-momentum polaritons in the same branch, and the only important contribution is the polariton to exciton scattering. Thus a very intriguing feature of this absorption model is the prediction that the disorder contribution to the polariton linewidth should disappear in structures with small but finite exciton inhomogeneous broadening, when polariton to exciton scattering becomes impossible because the polariton energies are too far in the tails of the excitonic absorption (see Fig. 3 in Ref. 5). More precisely, the disorder contribution to the polariton linewidth should disappear, especially in the lower polariton, when the exciton inhomogeneous broadening is much narrower than the Rabi splitting in the microcavity (weak-disorder limit). However, this point was not experimentally verified in these previous works, also due to the significant exciton inhomogeneous broadening in the samples investigated. Recent achievements in growth of high-quality microcavities ${ }^{6,7}$ now open the possibility of experimentally verifying this prediction.

In this article we report a detailed investigation of the polariton linewidths in a series of semiconductor microcavities with variable exciton inhomogeneous broadening, in the weak-disorder regime. We have used molecular beam epitaxy grown GaAs single QW's embedded in AlGaAs microcavities, with three different well widths of $25 \mathrm{~nm}, 15 \mathrm{~nm}$, and $10 \mathrm{~nm}$. With decreasing well width, the exciton becomes more sensitive to the interface and alloy disorder due to the larger wave function overlap with the interface region. The 


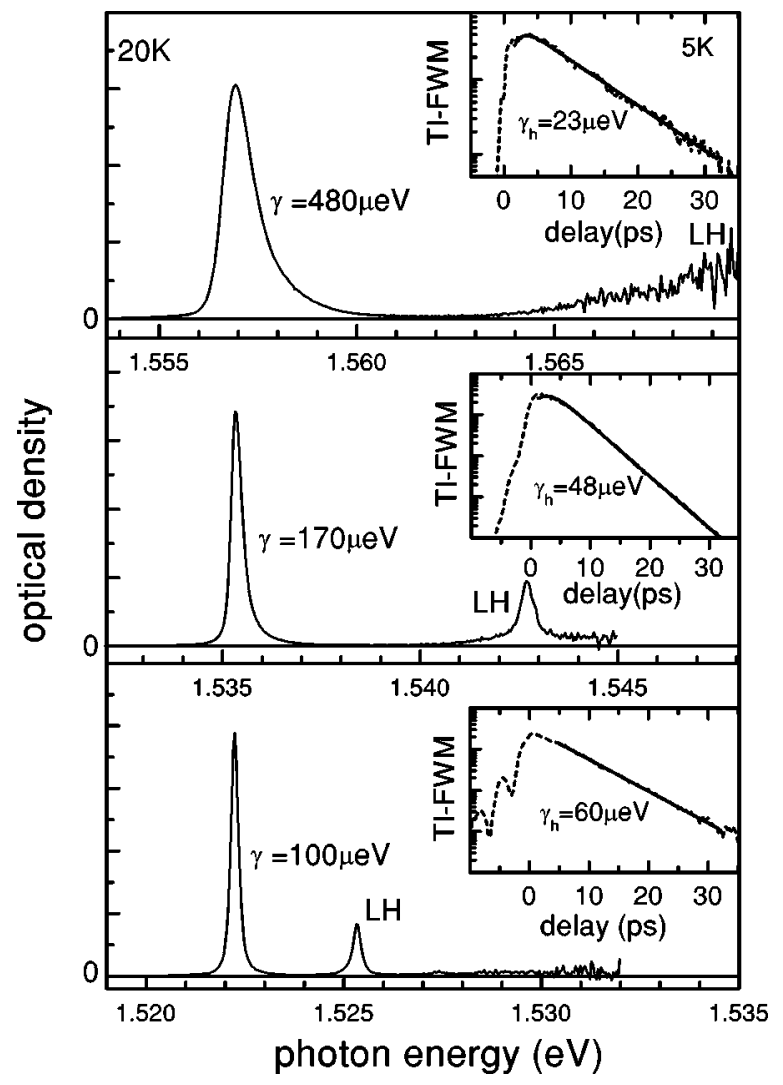

FIG. 1. Absorption profile, as deduced from the photoluminescence spectrum at $20 \mathrm{~K}$ corrected by a Boltzmann population factor, for a $25 \mathrm{~nm}$ (bottom), a $15 \mathrm{~nm}$ (middle), and a $10 \mathrm{~nm}$ (top) $\mathrm{GaAs} / \mathrm{Al}_{0.3} \mathrm{Ga}_{0.7} \mathrm{As}$ single quantum well. In the insets, four-wave mixing $(\mathrm{FWM})$ traces at $5 \mathrm{~K}$ are given with the corresponding fits of the homogeneous broadening.

optical properties of bare $\mathrm{GaAs} / \mathrm{Al}_{0.3} \mathrm{Ga}_{0.7} \mathrm{As}$ QW's of the same nominal thicknesses and grown under similar conditions are shown in Fig. 1. The excitonic absorption spectrum is deduced from the photoluminescence spectrum, measured at $20 \mathrm{~K}$ at resonant excitation and low intensity, divided by the Boltzmann population factor, assuming an exciton population in thermal equilibrium at the lattice temperature as described in Ref. 8. This procedure is already a good approximation at $20 \mathrm{~K}$ in the samples investigated as we have experimentally verified by comparison with higher temperatures. The corresponding half widths at half maximum (HWHM's) of the HH excitonic absorption are indicated. All the linewidths will be given in the following as HWHM's. In order to estimate the exciton inhomogeneous broadening we have compared these linewidths with measurements of the homogeneous broadening by degenerate four-wave mixing (FWM). Details of the experimental setup can be found in Ref. 9. In the inset, the time-integrated FWM for collinearly polarized pulses is shown versus their relative delay time at 5 $\mathrm{K}$ close to the low-density regime $\left(\sim 10^{9}\right.$ excitons $\left./ \mathrm{cm}^{2}\right)$. For the $25 \mathrm{~nm}$ wide QW (bottom plot) the decay is monoexponential and the FWM is a free-polarization decay, indicating the absence of disorder probed by the exciton. ${ }^{9}$ The corresponding homogeneous broadening agrees well with the absorption linewidth at $20 \mathrm{~K}$, when we take into account
$2.1 \mu \mathrm{eV} / \mathrm{K}$ linewidth broadening by acoustic-phonon absorption. ${ }^{9}$ Thus, the exciton absorption in the $25 \mathrm{~nm}$ QW is predominantly homogeneously broadened. In the $15 \mathrm{~nm}$ wide QW (middle plot) signatures of exciton inhomogeneous broadening appear in the absorption line shape, which is broader and slightly asymmetric. ${ }^{4}$ The FWM is now a photon echo with a delayed maximum, and its decay was fitted as proposed by Erland et al. ${ }^{10}$ An acoustic-phonon coefficient of $\sim 2 \mu \mathrm{eV} / \mathrm{K}$ was also measured in this $\mathrm{QW}$, resulting in a homogeneous broadening contribution of $\sim 80 \mu \mathrm{eV}$ at 20 $\mathrm{K}$. Thus the total linewidth of the $15 \mathrm{~nm}$ wide $\mathrm{QW}$ is an interplay of comparable homogeneous and inhomogeneous broadenings. Finally, the absorption profile of the $10 \mathrm{~nm}$ QW (top plot) is strongly inhomogeneously broadened, with a negligible contribution due to the homogeneous broadening as deduced by FWM, and largely asymmetric.

We have recently reported on the optical properties of a microcavity formed by embedding a $25 \mathrm{~nm}$ GaAs single QW inside an $\mathrm{AlGaAs} \lambda$ cavity. ${ }^{6,11} \mathrm{An} \mathrm{AlAs} / \mathrm{Al}_{0.15} \mathrm{Ga}_{0.85} \mathrm{As}$ Bragg reflector of 25 (16) periods was grown at the bottom (top) of the cavity. A special design of the $\mathrm{Al}_{0.3} \mathrm{Ga}_{0.7} \mathrm{As}$ barriers and the spacer layer was shown to be successful in reducing excess free-carrier densities in the microcavity compared to more conventional designs. ${ }^{11}$ We obtained a Rabi splitting to linewidth ratio of 19 at $11 \mathrm{~K}$, one of the best reported until now to our knowledge for single QW microcavities. With the same design, we have grown two new microcavities containing, respectively, a $15 \mathrm{~nm}$ and a $10 \mathrm{~nm}$ wide single GaAs QW, which are investigated in this work in comparison with the $\mathrm{MC}$ containing the $25 \mathrm{~nm} \mathrm{QW}$. The energy of the polaritons versus the energy distance between the cavity resonance and the bare $\mathrm{HH}$ exciton (detuning) is shown in Fig. 2 for these two microcavities at low temperature $(5 \mathrm{~K})$. A good fit to the data is obtained by solving a three-coupled-oscillator model including the light-hole (LH) exciton. ${ }^{11}$ The coupling to the LH exciton results in the appearance of three polariton modes, the lower, middle, and upper polaritons. From the fits we deduced $3.8 \mathrm{meV} \mathrm{HH}$ Rabi splitting (2.4 meV LH Rabi splitting) for the MC containing the $15 \mathrm{~nm} \mathrm{QW}$, and $4.15 \mathrm{meV}$ HH Rabi splitting (2.8 meV LH Rabi splitting) for the MC containing the $10 \mathrm{~nm}$ $\mathrm{QW}$. Thus, in all the microcavities investigated the $\mathrm{HH}$ exciton inhomogeneous broadening is smaller than one-half the HH Rabi splitting, i.e., the structures are in the weakdisorder regime. ${ }^{5}$ Note that for smaller well widths the stronger quantization of the $\mathrm{LH}$ exciton leads to a smaller mixing of this state in the LP and middle polariton (MP) resonances around zero detuning.

In the inset, the reflectivity spectrum measured with white light near normal incidence $\left(\sim 1^{\circ}\right)$ is shown for both MC's at zero detuning. The white-light source was focused on the sample with a spot diameter of $70 \mu \mathrm{m}$ resulting in a small energy broadening of the cavity mode from the wedge gradient and the incident wave vector spread. ${ }^{11}$ The spectra were detected using a spectrometer and an intensified multichannel analyzer, with a total spectral resolution close to a Lorentzian line shape of $65 \mu \mathrm{eV}$ HWHM. It can be seen from the insets of Fig. 2 that the LP linewidth is narrower 


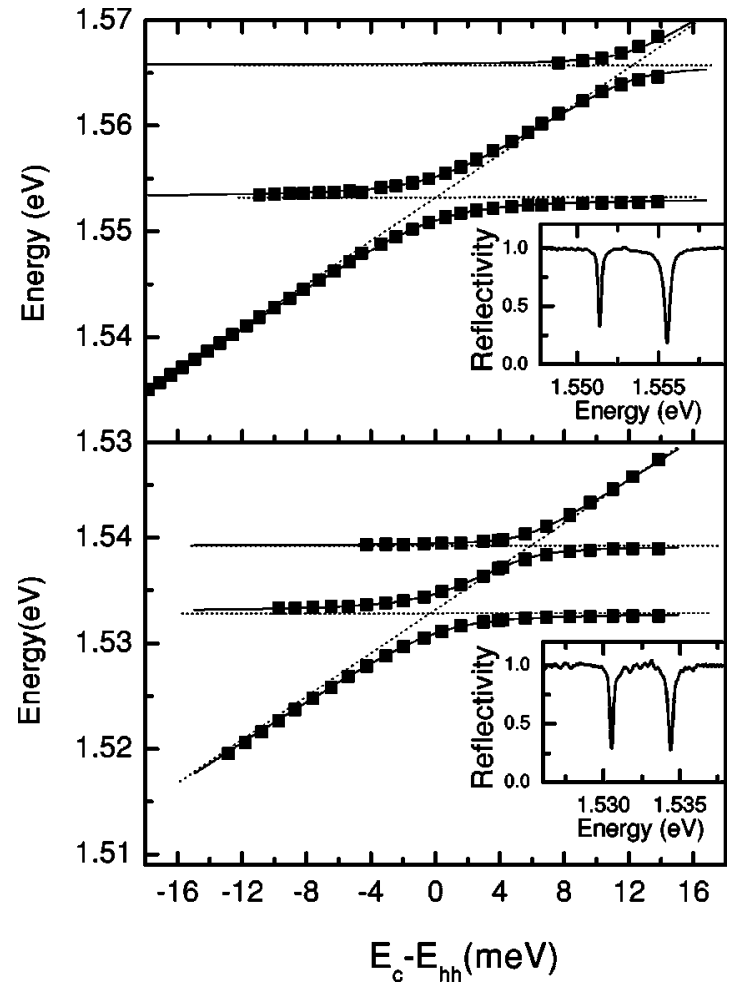

FIG. 2. Polariton energies versus detuning for the $10 \mathrm{~nm}$ (upper) and the $15 \mathrm{~nm}$ (lower) GaAs quantum well microcavity at $5 \mathrm{~K}$. The solid lines are fits to the data. The insets show the reflectivity spectrum of the microcavity at zero detuning.

than that of the MP and its value is subaverage, in agreement with previous observations on the effect of disorder on the polariton linewidths. ${ }^{1}$

In order to investigate in detail the role of the disorder in the polariton linewidths, we have measured the detuning dependence of the LP and MP linewidths, as shown in Fig. 3. The measured linewidths, well fitted with Lorentzian profiles, have been corrected by subtracting the spectral resolution. The closed squares are the LP linewidths while the open circles are the MP linewidths. The solid (dotted) lines are the cavity linewidths, due to the finite transmission of the Bragg mirrors, multiplied by the calculated photonic content in the lower (middle) polariton. The cavity linewidth was calculated taking into account the change of the linewidth with energy position in our specially designed structure with uniform Bragg mirrors and a wedged spacer layer, ${ }^{11}$ and agrees well with the measured LP linewidth at large negative detunings. Uncertainties in the fit of the cavity linewidths are less than $10 \%$. Comparable LP broadenings are measured at large negative detuning in all the samples, consistent with the fact that all the samples have nominally equal Bragg mirror reflectivities. It can be clearly seen from Fig. 3 that close to zero detuning the 15 and $10 \mathrm{~nm}$ QW MC's show a LP linewidth narrower than that of the MP. Moreover, from the comparison with the calculated photonic part the LP linewidth is subaverage and given only by the cavity contribution. In the $25 \mathrm{~nm}$ QW MC, instead, the LP and MP linewidths are almost equal at zero detuning, and the LP linewidth is larger than the cavity linewidth contribution.

In order to give a physical picture of the observed results,

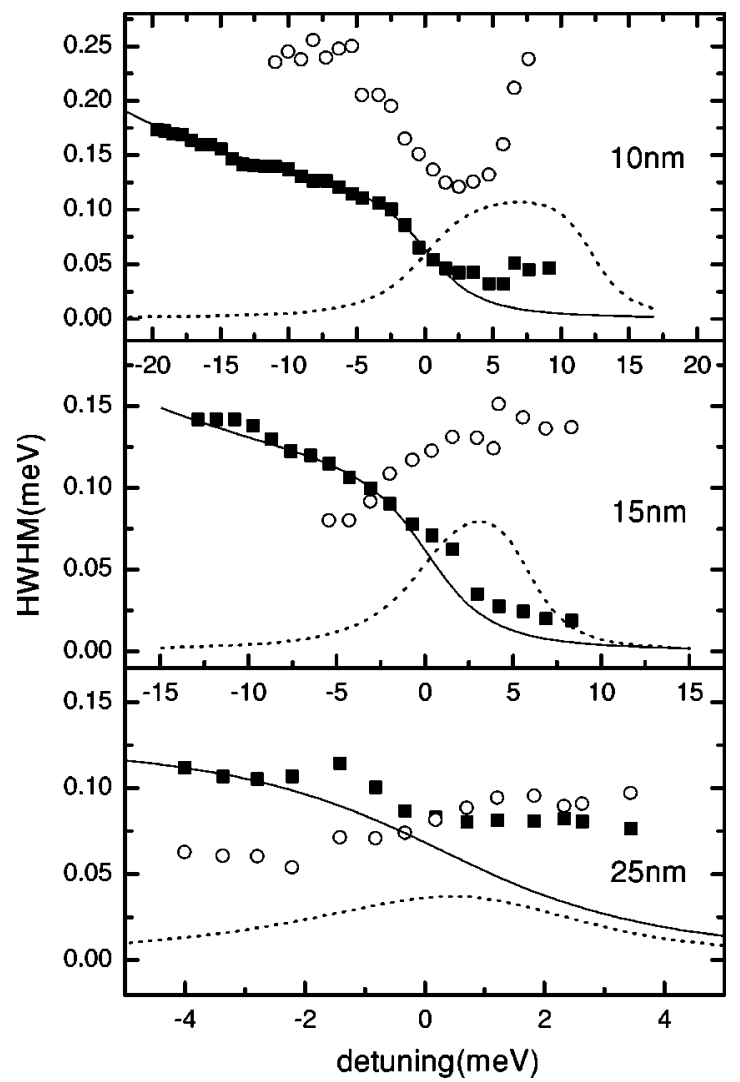

FIG. 3. Linewidths versus detuning of the lower (closed squares) and middle (open circles) polaritons, for the different microcavity samples as indicated. The solid (dotted) lines are the calculated photonic linewidths of the lower (middle) polaritons.

we have plotted in Fig. 4 the polariton linewidths versus the distance $E_{\mathrm{P}}-E_{\mathrm{HH}}$ of the polariton energy from that of the $\mathrm{HH}$ bare exciton. According to the simple absorption model of Whittaker, ${ }^{5}$ the polariton linewidth is the sum of the cavity contribution and an excitonic part. This excitonic part $\gamma_{e}$ of the linewidth should be related to the strength of the exciton absorption probed by the polariton resonances. We have quantitatively performed this comparison. The measured absorption spectra in Fig. 1 have been scaled in order to represent the absorption probability in the QW. ${ }^{12}$ The area of the absorption probability $\alpha(\omega)$ in a QW is proportional to the oscillator strength per unit $\operatorname{area}^{13} f$ :

$$
\int \alpha(\omega) d(\hbar \omega)=\frac{2 \pi^{2} \hbar e^{2}}{n m_{0} c} f
$$

with the refractive index $n$, the electron charge $e$ and mass $m_{0}$, and the speed of light $c$. The oscillator strength per unit area is proportional to the exciton radiative linewidth, ${ }^{13}$ which is related to the Rabi splitting $\hbar \Omega$ in the microcavity, ${ }^{14}$ and one gets

$$
f=\frac{(\hbar \Omega)^{2} n^{2} m_{0} L_{\mathrm{eff}}}{8 \pi e^{2} \hbar^{2}}
$$

with $L_{\text {eff }}$ the effective length in the microcavity due to the penetration length into the Bragg mirrors. The absorption probability is a loss in the cavity that gives rise to a photon 


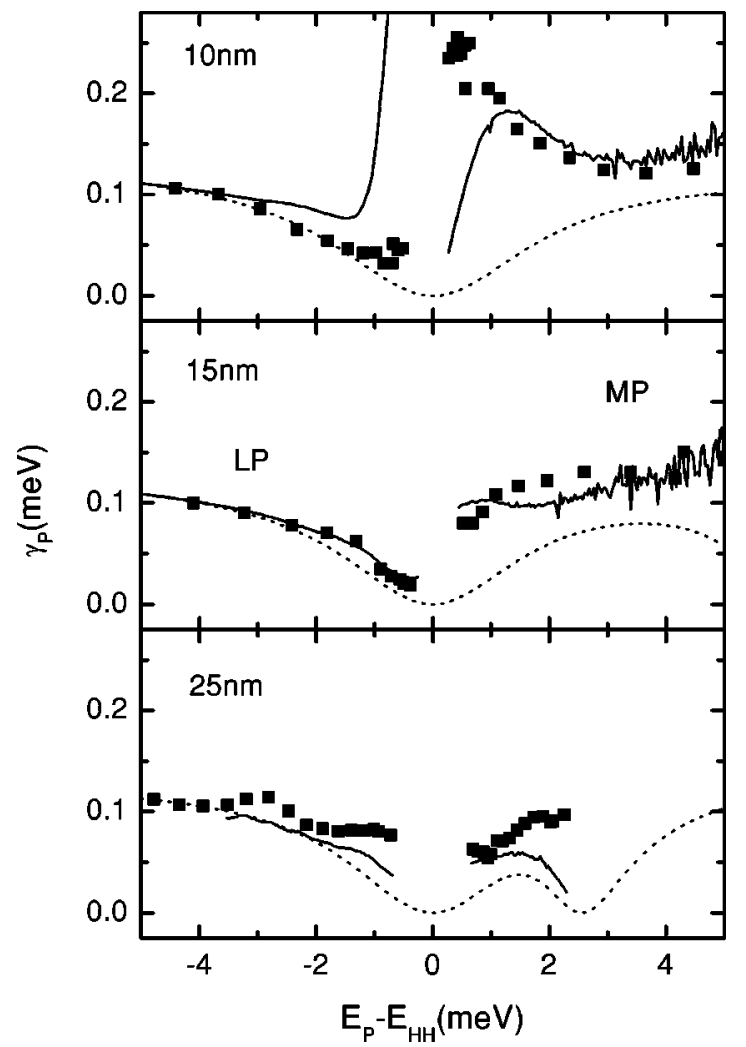

FIG. 4. Measured (symbols) and calculated (lines) polariton linewidths versus the energy distance of the polariton from the bare $\mathrm{HH}$ exciton. The dotted lines are the estimated cavity contributions in the polariton linewidths.

decay rate $\alpha(\omega) c\left(n L_{\text {eff }}\right)^{-1}$ additional to the transmission losses from the Bragg mirrors. Thus, finally, the measured absorption spectrum with an integral over the energy normalized to $1, s(\omega)$, is related to $\gamma_{e}$ by

$$
\gamma_{e}=\left|c_{l}\right|^{2} s(\omega) \frac{\pi(\hbar \Omega)^{2}}{8}
$$

with $\left|c_{l}\right|^{2}$ being the photonic content of the polariton at the energy $\hbar \omega$. This formula is a valid approximation when $\left|c_{l}\right|^{2}$ is not too small; otherwise it predicts that the polariton linewidth tends to zero when the photonic content tends to zero, while the true value tends to the exciton linewidth. ${ }^{5}$

The solid lines in Fig. 4 are the calculated polariton linewidths $\gamma_{\mathrm{P}}=\left|c_{l}\right|^{2} \gamma_{c}+\gamma_{e}$ with $\gamma_{c}$ the cavity linewidth (see Fig. 3) and $\gamma_{e}$ calculated using Eq. (3) and the absorption spectra in Fig. 1. For comparison, the isolated cavity linewidth contribution $\left|c_{l}\right|^{2} \gamma_{c}$ is plotted as dotted lines. The overall quantitative agreement between calculated and measured linewidths is quite good for $\left|E_{\mathrm{P}}-E_{\mathrm{HH}}\right|>1 \mathrm{meV}$, where the absorption model holds, i.e., when the photonic content is not too small and when the polariton energies are resonant with excitonic states far enough in the tail of the absorption to be in the weak-coupling regime. ${ }^{5}$ In particular, it appears clearly that when the excitonic absorption profile is asymmetric the contribution to the polariton linewidth from the excitonic absorption is bigger for $E_{\mathrm{P}}-E_{\mathrm{HH}}>0$, i.e., for the MP, than for $E_{\mathrm{P}}-E_{\mathrm{HH}}<0$, i.e., for the LP, in agreement with the measurements. Moreover, in the LP the disorder-induced broadening is absent when the polariton energies are far in the tail of the excitonic absorption.

Some small discrepancies between calculation and measurements are also evidenced. A parameter not under control in this comparison is the density of excess free carriers in the samples, resulting in trion resonances in the low-energy tail of the excitonic absorption. In the upper part of Fig. 4 a trion resonance $\sim 2 \mathrm{meV}$ below the exciton seems to be present in the $10 \mathrm{~nm}$ bare QW, which is not observed in the microcavity sample. The opposite occurs in the $25 \mathrm{~nm}$ QW (lower figure), where a trion resonance is evidenced in the microcavity sample, approximately $1 \mathrm{meV}$ below the $\mathrm{HH}$ exciton, also in agreement with photoluminescence measurements on this structure, ${ }^{11}$ while it is less present in the bare QW sample. Additionally, in the $15 \mathrm{~nm}$ and $25 \mathrm{~nm}$ QW MC's, the MP linewidths appear to be slightly higher (by a few tens of $\mu \mathrm{eV}$ ) than the calculated ones in the region where the photonic content is not very small. Even if this difference is at the limit of our accuracy, it is reasonable that when the disorder-induced broadening is very small the MP linewidths are affected by other scattering processes, such as phonon or free-carrier interactions, not included in the simple absorption model of Whittaker. ${ }^{5}$ In fact, the observed difference is on the order of what is calculated from additional homogeneous broadening of the upper polariton due to acoustic phonon scattering, ${ }^{15}$ which is inhibited in the lower polariton. As a general comment, note that the polariton linewidths shown in this work are very small, well below those reported in previous work investigating the role of disorder in cavity polaritons. ${ }^{3,5}$ Thus it is not surprising that small features such as trion/free carriers and phonon effects are affecting the measured linewidths in our samples.

In conclusion, we have shown experimentally that the influence of disorder on the polariton linewidth of microcavities in the weak-disorder regime can be canceled when the polariton energies are far in the tail of the excitonic absorption, in agreement with predictions in the literature. ${ }^{5,2}$ The measured linewidths have been quantitatively compared with those estimated from the measured excitonic absorption in the bare quantum wells, following the simple absorption model proposed by Whittaker. ${ }^{5}$ A generally good agreement is found.
${ }^{1}$ D.M. Whittaker, P. Kinsler, T.A. Fisher, M.S. Skolnick, A. Armitage, A.M. Afshar, M.D. Sturge, and J.S. Roberts, Phys. Rev. Lett. 77, 4792 (1996).

${ }^{2}$ V. Savona, C. Piermarocchi, A. Quattropani, F. Tassone, and P. Schwendimann, Phys. Rev. Lett. 78, 4470 (1997).
${ }^{3}$ C. Ell, J. Prineas, T.R. Nelson, S. Park, H.M. Gibbs, G. Khitrova, S.W. Koch, and R. Houdré, Phys. Rev. Lett. 80, 4795 (1998).

${ }^{4}$ R.F. Schnabel, R. Zimmermann, D. Bimberg, H. Nickel, R. Lösch, and W. Schlapp, Phys. Rev. B 46, 9873 (1992).

${ }^{5}$ D.M. Whittaker, Phys. Rev. Lett. 80, 4791 (1998). 
${ }^{6}$ P. Borri, J.R. Jensen, W. Langbein, and J.M. Hvam, Phys. Rev. B 61, R13 377 (2000).

${ }^{7}$ M. Saba, F. Quochi, U. Oestersle, J.L. Staehli, B. Deveaud, G. Bongiovanni, and A. Mura, Phys. Status Solidi A 178, 149 (2000).

${ }^{8}$ M. Gurioli, A. Vinattieri, J. Martinez-Pastor, and M. Colocci, Phys. Rev. B 50, 11817 (1994).

${ }^{9}$ W. Langbein and J.M. Hvam, Phys. Rev. B 61, 1692 (2000).

${ }^{10}$ J. Erland, K.H. Pantke, V. Mizeikis, V.G. Lyssenko, and J.M. Hvam, Phys. Rev. B 50, 15047 (1994).

${ }^{11}$ J.R. Jensen, P. Borri, W. Langbein, and J.M. Hvam, Appl. Phys. Lett. 76, 3262 (2000).

${ }^{12}$ Note that, from the comparison of the bare HH energy positions measured in the spectra of Fig. 1 and deduced by the fits of the polariton energies in Fig. 2, we actually found a small deviation in the effective well width inside the microcavity, which is $\sim 5 \%$ larger compared to the bare sample. Thus the absorption spectra have been corrected by rescaling the energy axis in order to fit the exciton quantization energy of the quantum well in the microcavity sample.

${ }^{13}$ L. C. Andreani, in Confined Electrons and Photons: New Physics and Applications, Vol. 340 of NATO Advanced Study Institute, Series B: Physics, edited by E. Burstein and C. Weisbuch (Plenum Press, New York, 1995), pp. 57-112.

${ }^{14}$ M.S. Skolnick, T.A. Fisher, and D.M. Whittaker, Semicond. Sci. Technol. 13, 645 (1998).

${ }^{15}$ V. Savona and C. Piermarocchi, Phys. Status Solidi A 164, 45 (1997). 